\title{
Admissible Large Perturbations in Structural Redesign
}

\author{
Michael M. Bernitsas* and Byungsik Kang† \\ University of Michigan, Ann Arbor, Michigan 48109
}

\begin{abstract}
A new algorithm for structural redesign by perturbation is developed. It allows for large changes between baseline and objective structures and can satisfy both modal and static displacement objectives. It is faster and more accurate than previously developed perturbation resizing methods because it advances incrementally to the objective structure using large admissible perturbations. The computer code developed is used as postprocessor to general or special purpose finite element codes, to improve upon the design of structures with unacceptable modal and/or static displacement response. Depending on the relation between the number of redesign goals, allowable structural changes, and admissibility constraints, the desired redesign may be feasible-either locally optimal or unique-or may not exist. In the latter case, a minimum-error inadmissible design is produced. Several numerical examples are used to study the effects of definition and relaxation of the redesign admissible domain and demonstrate the accuracy of the new redesign algorithm. An offshore tower with repeated eigenvalues and 192 degrees of freedom is also redesigned subject to frequency, static displacement goals, and admissibility constraints.
\end{abstract}

$c_{i j}$

\section{E}

$\{f\}, f_{j}$

$\left[k_{e}\right]$

$[k],[` K \backslash$

$K_{i}$

$\ell$

$\left[m_{e}\right]$

$[m],\left[{ }^{\prime} M_{\backslash}\right]$

$M_{i}$

$n$

$N$

$n_{r}$

$p$

$q$

\section{Nomenclature}

= admixture coefficient for participation of the $j$ th mode to changes in the $i$ th mode

$=$ Young's modulus

$=$ nodal force vector and its $j$ th component

$=$ stiffness matrix of element or group of elements related to property $e$

$=$ global and generalized stiffness matrices

$=i$ th component of [ $K \backslash]$

$=$ increment number

$=$ mass matrix of element or group of elements related to property $e$

$=$ global and generalized mass matrices

$=i$ th component of [ $\left.M_{\backslash}\right]$

$=$ number of degrees of freedom of structural model

$=$ number of increments in predictor-corrector algorithm

$=$ number of baseline structural modes used in redesign

$=$ number of redesign variables

$=$ previous increments in increment $\ell$; $q=1,2, \ldots,(\ell-1)$

RESTRUCT $=$ program for REdesign of STRUCTures

$S, S_{a}, S_{e}, S_{i}=$ number of redesign, admissibility, equality, and inequality redesign constraints, respectively

$S_{b} \quad=$ number of upper and lower bound inequality constraints imposed on $\alpha_{e}$

$S_{u}, S_{\omega}, S_{\phi} \quad=$ number of displacement, frequency, and modal node redesign constraints, respectively

$\{u\}$

$=$ nodal static displacement vector

Indices and Special Symbols

$\ell \quad=$ index denoting quantity in increment $\ell$; $\ell=1,2, \ldots, N$

Received June 29, 1989; revision received Jan. 29, 1990. Copyright (C) 1990 by the American Institute of Aeronautics and Astronautics, Inc. All rights reserved.

*Associate Professor, Department of Naval Architecture and Marine Engineering.

†Graduate Student, Research Assistant, Department of Naval Architecture and Marine Engineering.

\begin{tabular}{|c|c|}
\hline $\begin{array}{l}\left.{ }^{\prime}{ }^{\prime}\right] \\
()^{\prime}\end{array}$ & $\begin{aligned}= & \text { diagonal matrix notation } \\
= & \text { symbol denoting objective structural } \\
& \text { property }\end{aligned}$ \\
\hline \multicolumn{2}{|c|}{ Greek Symbols } \\
\hline$\alpha_{e}$ & $\begin{array}{l}=\text { fractional change to element or group } \\
\text { property } e\end{array}$ \\
\hline$\delta$ & $\begin{array}{l}=\text { prefix denoting incremental change to } \\
\text { structural quantities }\end{array}$ \\
\hline$\Delta$ & $\begin{array}{l}=\text { prefix denoting total change to structural } \\
\text { quantities }\end{array}$ \\
\hline$[\phi]$ & $=$ matrix of mode shape vectors \\
\hline$\{\Psi\}_{i}$ & $=i$ th mode shape \\
\hline & $=k$ th degree of freedom of $i$ th mode shape \\
\hline$\omega_{i}$ & $=i$ th natural frequency \\
\hline
\end{tabular}

\section{Introduction}

Q TRUCTURAL design of large-scale structures usually $\checkmark$ cannot be achieved in one step. A design spiral process may be necessary to satisfy all design goals and produce a structure with satisfactory response. In case the shape of a structure has been defined to satisfy functional and operational requirements, the structural design process is schematically shown in Fig. 1. This involves finite element (FE) modeling, FE analysis by general or special purpose codes, and redesign, which in this paper has the meaning of resizing. ${ }^{1}$ Resizing is defined as the process of altering cross-sectional properties of elements in a FE model where grid points are fixed. Reshaping - not considered in this work - would involve alteration of grid topology. Redesign by trial and error is time consuming, expensive, and occasionally inconclusive. Sensitivity and perturbation methods have been developed in order to automate the redesign process.

Sensitivity methods that have been extensively studied by Haug et al..$^{2,3}$ are appropriate for small structural changes, and are especially efficient when analytical sensitivity expressions are available. Perturbation-based redesign methods have been developed in the past fifteen years and presently can solve large-scale redesign problems allowing for large structural changes. Linear perturbation methods, allowing for small structural changes, were introduced by Stetson et al. ${ }^{4-7}$ and later improved by Sandström et al. ${ }^{8,9}$ Recently, two different approaches to nonlinear perturbation allowing for large structural and response changes have been developed. The first 


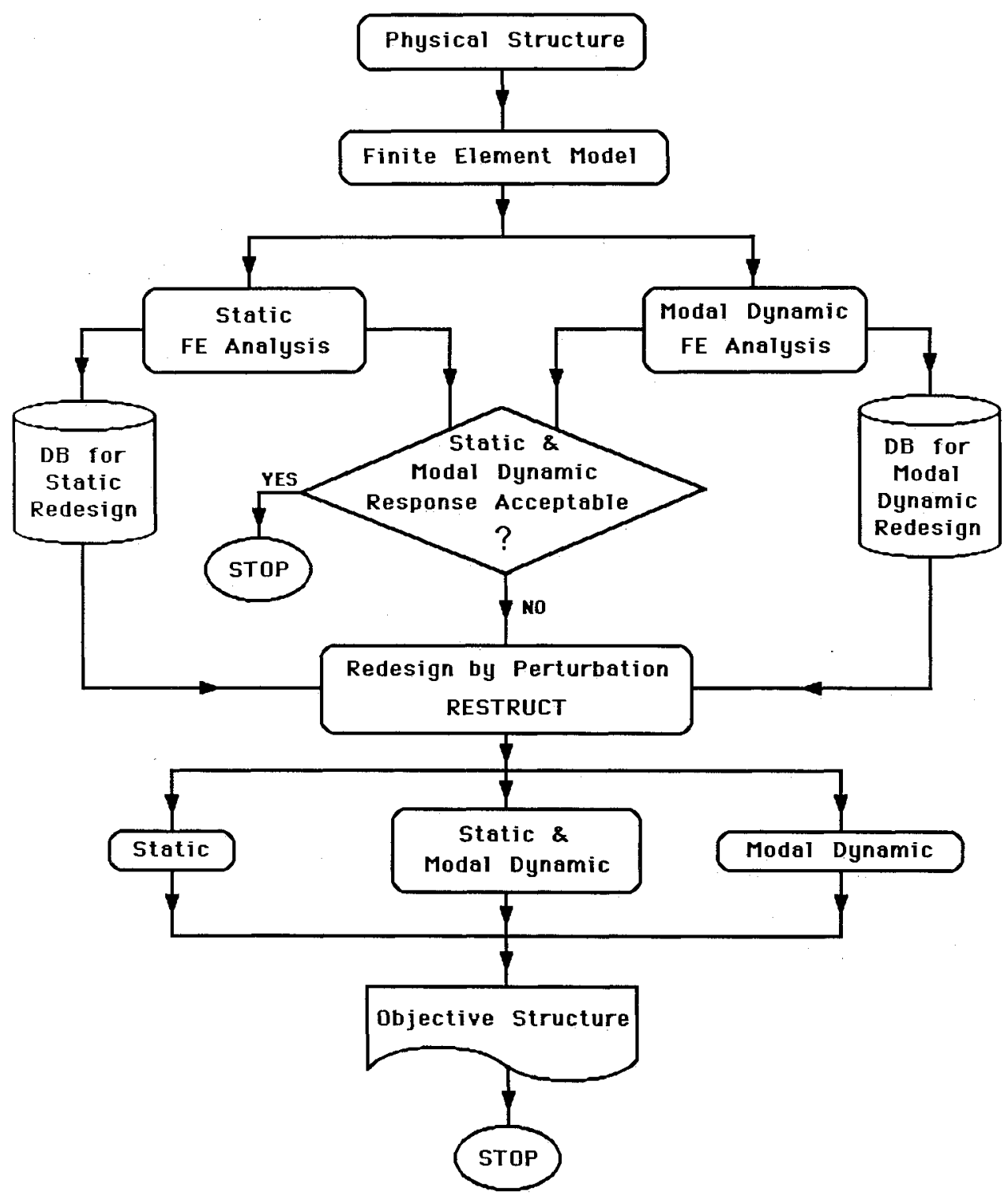

Fig. 1 Procedure for structural design, modeling, analysis, and redesign.

approach, developed by Kim and Anderson, ${ }^{10}$ used a penalty method to satisfy modal dynamic objectives. It is theoretically exact but its applicability is limited to small-scale structures due to the high cost and limited accuracy of the adopted nonlinear search technique. Dynamic condensation was applied to reduce the high cost of the penalty method for large-scale structures. ${ }^{11}$ The second approach treats larger changes in the redesign goals incrementally. In each increment, a predictorcorrector scheme is employed, where in the predictor phase Stetson's linear method is used. Hoff et al. ${ }^{12}$ and Hoff and Bernitsas ${ }^{13,14}$ introduced modal objectives (both frequency and mode) in the redesign process; Bernitsas et al. ${ }^{15}$ included variable tension geometric stiffness matrices in dynamic redesign of marine risers; Gans and Anderson ${ }^{16}$ incorporated centrifugal and Coriolis effects; Hoff and Bernitsas ${ }^{17}$ redesigned for static objectives; Kim and Bernitsas ${ }^{18,19}$ developed an integrated method to redesign for both modal and static displacement objectives simultaneously. It should be emphasized that the second approach can be used to redesign a structure to change its natural frequencies and modify its mode shapes, ${ }^{17}$ and change its static response ${ }^{18,19}$ as well. Furthermore, redesign techniques must not be confused with reanalysis method. ${ }^{1,20}$

The incremental predictor-corrector approach is more powerful, accurate, and capable of solving larger scale redesign problems allowing at the same time for larger changes to response particulars. Recently, the trend in research has been to include new finite elements and stiffness matrices ${ }^{15,16}$ and redesign for more diverse objectives. ${ }^{18}$ Presently, an effort is being made to improve the accuracy of incremental predictor-corrector schemes for strongly nonlinear problems. This would allow for solution of larger scale problems, larger changes in the redesign objectives, and possibly use of larger steps in the process of advancing incrementally from baseline to objective structure. Thus, Gans and Anderson ${ }^{16}$ introduced a FE reanalysis by MSC/NASTRAN at the end of both predictor and corrector phases in each increment. This approach, however, violates one of the fundamental principles of redesign by perturbation which is to find the objective structure using results of a single FE analysis, namely that of the baseline structure. In this paper, a new incremental predictor-corrector algorithm is developed, which allows for large structural changes, can satisfy both modal and static displacement redesign goals, and requires only one FE analysis-that of the baseline structure. This is achieved by ascertaining that at the end of each increment, the design obtained satisfies modal orthogonality conditions and therefore represents a real structure. In terms of structural perturbations, it implies that perturbations are admissible.

The problem of redesign by perturbation to meet modal and 
static displacement constraints is briefly presented in Sec. II. The new algorithm that identifies an objective design by large admissible perturbations is developed in Sec. III. Numerical implementation of the new algorithm in code RESTRUCT (REdesign of STRUCTures) is also discussed. Finally, numerical applications are used in order to compare the new algorithm to previous ones and investigate the significance of the number of admissibility constraints and the accuracy to which they are satisfied by the objective structure.

\section{Structural Redesign by Perturbation}

The structural design/modeling/analysis/redesign process depicted in Fig. 1 shows that redesign is performed if the results of static and/or modal dynamic FE analyses are not satisfactory. The equations of static and free vibration equilibrium of the descritized baseline structure are

$$
\begin{gathered}
{[k]_{n \times n}\{u\}_{n \times 1}=\{f\}_{n \times 1}} \\
\left([k]-\omega_{j}^{2}[m]\right)\{\psi\}_{j}=\{0\} \quad \text { for } j=1,2, \ldots, n
\end{gathered}
$$

where the $n$ eigenvalues $\omega_{j}, j=1,2, \ldots, n$, satisfy Eq. (3);

$$
\operatorname{det}\left([k]-\omega^{2}[m]\right)=0
$$

In Eq. (2), damping can be included only in Rayleigh's form. In matrix form, the $n$ free vibration equilibrium Eq. (2) can be written as

$$
[k][\phi]=[m][\phi]\left[{ }^{\prime} \omega^{2} \backslash\right]
$$

where $[\phi]=\left[\{\psi\}_{1},\{\psi\}_{2}, \ldots,\{\psi\}_{n}\right]$, and $\left[{ }^{\prime} \omega^{2}\right]$ is the diagonal matrix of the eigenvalues. Premultiplying Eq. (4) by $[\phi]^{T}$, we obtain the uncoupled modal equations in the following matrix form:

$$
\left[K_{\backslash}\right]=\left[{ }^{`} M \backslash\right]\left[\omega^{\prime}{ }^{2}\right]
$$

where [ $K \backslash$ ] and [ $\left.M_{\backslash}\right]$ are the generalized stiffness and mass matrices. Equations (1-5) also hold for the objective structure. All quantities related to the latter are hereafter primed. The following perturbation relations between baseline and objective structures are introduced:

$$
\begin{gathered}
{\left[k^{\prime}\right]=[k]+[\Delta k]} \\
\left\{u^{\prime}\right\}=\{u\}+\{\Delta u\} \\
{\left[m^{\prime}\right]=[m]+[\Delta m]} \\
{\left[{ }^{\prime} \omega^{\prime 2} \backslash\right]=\left[{ }^{\prime} \omega^{2} \backslash\right]+\left[{ }^{\prime} \Delta\left(\omega^{2}\right) 、\right]} \\
{\left[\phi^{\prime}\right]=[\phi]+[\Delta \phi]}
\end{gathered}
$$

where prefix $\Delta$ indicates change to a baseline quantity. Furthermore, assume that $p$ properties of elements or groups of elements are allowed to change and let $\alpha_{e}$ be the fractional change in property $e$. More than one property may be allowed to change in each element or group of elements. Expressing change in a global matrix as sum of all changes, we derive

$$
\begin{gathered}
{[\Delta k]=\sum_{e=1}^{p}\left[\Delta k_{e}\right]=\sum_{e=1}^{p}\left[k_{e}\right] \alpha_{e}} \\
{[\Delta m]=\sum_{e=1}^{p}\left[\Delta m_{e}\right]=\sum_{e=1}^{p}\left[m_{e}\right] \alpha_{e}}
\end{gathered}
$$

where several $\alpha_{e}$ may refer to the same element but different properties like bending, torsion, stretching etc., and some $\alpha_{e}$ may not represent fractional change of only $\left[k_{e}\right]$ or $\left[m_{e}\right]$. Assuming that $\{f\}=\left\{f^{\prime}\right\}$, i.e., that the nodal static force vector is the same for the baseline and objective structures, and inserting perturbation Eqs. (6) and (7) and Eqs. (11) and (12) in Eq. (1), we derive the general static perturbation Eq. $(13)^{18}$ :

$$
\{\Delta u\}=-\sum_{e=1}^{p}\left([k]+\sum_{e=1}^{p}\left[k_{e}\right] \alpha_{e}\right)^{-1}\left[k_{e}\right]\{u\} \alpha_{e}
$$

Similarly, inserting perturbation Eqs. (6), (8), (9), (10), and Eqs. (11) and (12) into the counterpart of Eq. (5) for the objective structure, we derive the general modal perturbation equations in scalar form ${ }^{18}$ :

$$
\begin{gathered}
\sum_{e=1}^{p}\left(\left\{\psi^{\prime}\right\}_{j}^{T}\left[k_{e}\right]\left\{\psi^{\prime}\right\}_{i}-\omega_{i}^{\prime 2}\left\{\psi^{\prime}\right\}_{j}^{T}\left[m_{e}\right]\left\{\psi^{\prime}\right\}_{i}\right) \alpha_{e} \\
=\left\{\psi^{\prime}\right\}_{j}^{T}[m]\left\{\psi^{\prime}\right\}_{i} \omega_{i}^{\prime 2}-\left\{\psi^{\prime}\right\}_{j}^{T}[k]\left\{\psi^{\prime}\right\}_{i}
\end{gathered}
$$

for $i, j=1,2, \ldots, n$. In addition, the objective structure modes $\left\{\psi^{\prime}\right\}_{j}, j=1,2, \ldots, n$, must be orthogonal with respect to $\left[k^{\prime}\right]$ and $\left[m^{\prime}\right]$. Theoretically, orthogonality of modes with respect to one of $\left[k^{\prime}\right]$ or $\left[m^{\prime}\right]$ implies orthogonality with respect to the other. Numerically, however, both conditions must be forced if $\left\{\psi^{\prime}\right\}_{j}, j=1,2, \ldots, n$, are to represent modes of a real structure. Thus, the $n^{2}$ scalar Eqs. (14) become

$$
\begin{aligned}
& \sum_{e=1}^{p}\left(\left\{\psi^{\prime}\right\}_{i}^{T}\left[k_{e}\right]\left\{\psi^{\prime}\right\}_{i}-\omega_{i}^{\prime 2}\left\{\psi^{\prime}\right\}_{i}^{T}\left[m_{e}\right]\left\{\psi^{\prime}\right\}_{i}\right) \alpha_{e} \\
& =\omega_{i}^{\prime 2}\left\{\psi^{\prime}\right\}_{i}^{T}[m]\left\{\psi^{\prime}\right\}_{i}-\left\{\psi^{\prime}\right\}_{i}^{T}[k]\left\{\psi^{\prime}\right\}_{i} \quad i=1,2, \ldots, n
\end{aligned}
$$

$$
\begin{aligned}
& \sum_{e=1}^{p}\left\{\psi^{\prime}\right\}_{j}^{T}\left[k_{e}\right]\left\{\psi^{\prime}\right\}_{i} \alpha_{e}=-\left\{\psi^{\prime}\right\}_{j}^{T}[k]\left\{\psi^{\prime}\right\}_{i} \\
& \sum_{e=1}^{p}\left\{\psi^{\prime}\right\}_{j}^{T}\left[m_{e}\right]\left\{\psi^{\prime}\right\}_{i} \alpha_{e}=-\left\{\psi^{\prime}\right\}_{j}^{T}[m]\left\{\psi^{\prime}\right\}_{i}
\end{aligned}
$$

for $i=1,2, \ldots, n$ and $j=i+1, i+2, \ldots, n$.

\section{Structural Perturbations and Redesign Variables}

The purpose of redesign is to produce structural perturbation quantities $[\Delta k]$ and $[\Delta m]$ as defined in Eqs. (6) and (8). Those are in general large and cannot be computed using sensitivity methods. The designer can run code RESTRUCT, which implements the algorithm developed in the next section, to compute perturbations $[\Delta k]$ and $[\Delta m]$ in order to satisfy certain modal dynamic and static displacement goals. The desired perturbations are expressed in terms of fractional changes of sectional linear dimensions (width, height) or properties (area, moment of inertia) by Eqs. (11) and (12). The designer has to decide which of those sectional properties of elements or groups of elements he/she would allow to change. Those fractional changes become redesign variables $\alpha_{e}$, $e=1,2, \ldots, p$, which are the unknown quantities in the redesign process. The latter must be computed to satisfy the following design goals.

\section{Design Goals and Response Constraints}

In terms of the $p, \alpha_{e}$ the perturbations in the structural response are expressed by Eq. (13) for $\{\Delta u\}$, Eq. (15a) for $\left\{\Delta\left(\omega^{2}\right)\right\}$, and Eqs. (15b) and (15c) for $[\Delta \phi]=\left[\{\Delta \phi\}_{1}\right.$, $\left.\{\Delta \phi\}_{2}, \ldots,\{\Delta \phi\}_{n}\right]$. Design goals may result in response constraints as follows. In the algorithm developed in this paper, the design goals below can be satisfied:

$$
\begin{array}{cc}
u_{i}^{\prime}=u_{i}+\Delta u_{i} \lesseqgtr b_{u_{i}}, & i=1,2, \ldots, S_{u} \\
\omega_{i}^{\prime 2}=\omega_{i}^{2}+\Delta \omega_{i}^{2} \lesseqgtr b_{\omega_{i}}, & i=1,2, \ldots, S_{\omega} \\
\phi_{k i}^{\prime}=\phi_{k i}+\Delta \phi_{k i} \lesseqgtr b_{\phi_{k i}}, & \text { number of }(k, i)=S_{\phi}
\end{array}
$$

where the right-hand sides $b_{u_{i}}, b_{\omega_{i}}, b_{\phi_{k i}}$ are the design goals to be specified by the designer; and $S_{u}, S_{\omega}$, and $S_{\phi}$ are the number 
of design goals imposed on the objective structure's response $\left\{u^{\prime}\right\}$, $\left[\omega^{2}\right]$, and $\left[\phi^{\prime}\right]$. Since the response of the baseline structure has been computed-by the one and only FE analysis performed in the redesign process-quantities $\{u\},\left[\omega^{2}\right]$ and $[\phi]$ are known. Thus, the design goals specified by Eqs. (16-18) result in constraints imposed on the desired response of the objective structure. Those constraints along with general perturbation Eqs. (13) and (15a-15c) produce constraints on redesign variables $\alpha_{e}$.

\section{Orthogonality Conditions and Admissibility Constraints}

The response constraints specified above-if satisfied in the solution process-will not necessarily guarantee that the structure corresponding to the computed $\alpha_{e}$ is real. To accomplish this, we have to make sure that not only the energy balance Eqs. (15a), which are related to the $S_{\omega}$ response constraints of Eq. (17), are satisfied, but that the $(n-1)$ orthogonality conditions corresponding to each of $S_{\omega}$ constraints are satisfied as well. This will ensure that the eigenpairs

$$
\left(\omega_{i}^{\prime},\left\{\psi^{\prime}\right\}_{i}\right), \quad i=1,2, \ldots, S_{\omega}
$$

can represent properties of a real structure. Since the objective structure, or any approximation to it, computed in the redesign process is not reanalyzed by a FE code, it is necessary to enforce the aforementioned orthogonality conditions on the modal perturbations $[\Delta \phi]$. Combining Eqs. (19), (15b), and $(15 \mathrm{c})$, we derive the following admissibility conditions for the objective structure:

$$
\begin{aligned}
& \sum_{e=1}^{p}\left\{\psi^{\prime}\right\}_{j}^{T}\left[k_{e}\right]\left\{\psi^{\prime}\right\}_{i} \alpha_{e}=-\left\{\psi^{\prime}\right\}_{j}^{T}[k]\left\{\psi^{\prime}\right\}_{i} \\
& \sum_{e=1}^{p}\left\{\psi^{\prime}\right\}_{j}^{T}\left[m_{e}\right]\left\{\psi^{\prime}\right\}_{i} \alpha_{e}=-\left\{\psi^{\prime}\right\}_{j}^{T}[m]\left\{\psi^{\prime}\right\}_{i}
\end{aligned}
$$

for $i=1,2, \ldots, S_{\omega}$ and $j=i+1, i+2, \ldots, n$. Equations (20) and (21) represent admissibility constraints on the redesign variables $\alpha_{e}$.

\section{Problem Definition}

The problem of redesign by perturbation can now be defined as follows: Calculate the values of redesign variables $\alpha_{e}$, $e=1,2, \ldots, p$, subject to $S_{u}$ static displacement constraints of Eq. (16), $S_{\omega}$ natural frequency constraints of Eq. (17), $S_{\phi}$ modal node constraints of Eq. (18), and

$$
2 \sum_{i=1}^{S_{\omega}}(n-i)=S_{\omega}\left[(2 n-1)-S_{\omega}\right]
$$

admissibility constraints of Eqs. (20) and (21).

The redesign problem is called static, dynamic, or integrated static/dynamic if only static, only dynamic, or both static and dynamic constraints are imposed on the redesign variables $\alpha_{e}$. In the last two versions of the design problem, admissibility constraints must be imposed on the redesign variables. For static redesign, a different algorithm has been developed. ${ }^{17}$

Even though the designer can specify only modal and static displacement goals for his/her objective structure, he/she can solve a plethora of redesign problems such as. 1) changing the natural frequencies of a structure to shift them out of the range of frequencies of excitation, 2) changing the predominant response normal mode to reduce excitation in a fluid-structure interaction problem, 3) improving fatigue particulars of a structure, and 4) reducing static stresses by controlling static displacements. Further improvements of the solution algorithm may solve directly the problem of redesigning for static stresses. Of higher priority, however, as considered by the authors are the problems of model correlation, redundancy analysis, and failure-mode identification that can be solved by RESTRUCT ${ }^{18}$ and will be studied in the near future.

\section{Solution by Large Admissible Perturbations}

The problem of redesign by perturbation, as defined in the previous section, requires that an objective structure be identified in the feasible domain defined by $S$ equality and inequality constraints, where

$$
S=S_{u}+S_{\omega}+S_{\phi}+S_{\alpha}
$$

$S_{a}$ is the number of admissibility constraints

$$
S_{a}=S_{\omega}\left[(2 n-1)-S_{\omega}\right]
$$

by $p$ lower bound inequality constraints on the $\alpha_{e}$

$$
-1<\alpha_{e}, \quad e=1,2, \ldots, p
$$

which are necessary to ensure that no negative property elements are generated; and by $2 p$ practical upper and lower bounds

$$
-1<\alpha_{e}^{-} \leq \alpha_{e} \leq \alpha_{e}^{+}, \quad e=1,2, \ldots, p
$$

The redesign problem has $p$ variables $\alpha_{e}$. The feasible domain 1) may be null, in which case no solution exists; 2) may have finite number of solutions, which is unlikely to occur in practical problems; and 3) or may be continuous in which case infinite acceptable redesigns exist. In the first case, RESTRUCT will produce a minimum-error solution in satisfaction of the $S+2 p$ constraints that define the feasible domain and will inform the user accordingly. In the other two cases, and especially in the last one, an optimization criterion is required to select the best of all possible redesigns. The criterion of minimum change between baseline and objective structures, used in this paper is

$$
\min \sum_{e=1}^{p} \alpha_{e}^{2}
$$

Another criterion often used in redesign is that of minimum weight for the objective structure. ${ }^{14,15,17,18}$ Selection of optimality criterion depends on the specific redesign application considered. For example, in the design process where a feasible design is sought, it is reasonable to use a minimum-weight criterion. For an existing structure, where improvement in performance is sought in a redesign process, it is preferable to use a minimum change criterion. In correlation between a real structure and its FE model-which can also be performed by perturbation-it is practical to select a minimum change criterion and impose additional constraints on the objective structure; e.g., a constant mass if the designer has confidence in the measurement of structural mass.

The difficulty of the problem can be recognized by looking at the complexity of the general static and dynamic perturbation Eqs. (13) and (15a), and the admissibility constraints of Eqs. (20) and (21) which along with inequalities of Eqs. (16-18) define the boundaries of feasible domain in implicit form in $\alpha_{e}$. Those boundaries depend on the modes of the objective structure, which depend implicitly on the unknown $\alpha_{e}$. The problem is resolved using an incremental predictorcorrector scheme that was developed in Refs. 18 and 19 for the integrated static/dynamic redesign problem and is modified below to ensure that incremental perturbations are admissible. The algorithm described below is summarized in Fig. 2.

\section{Incremental Perturbations}

All redesign objectives that are defined by the right-hand sides of inequalities of Eqs. (16-18) are achieved in an incremental approach, where in each increment no more than a $7 \%$ change in the objective is allowed. Let prefix $\delta$ denote changes within each increment $\ell, \ell=1,2, \ldots, N$. Then, the fully nonlinear incremental redesign problem is defined in the most general 


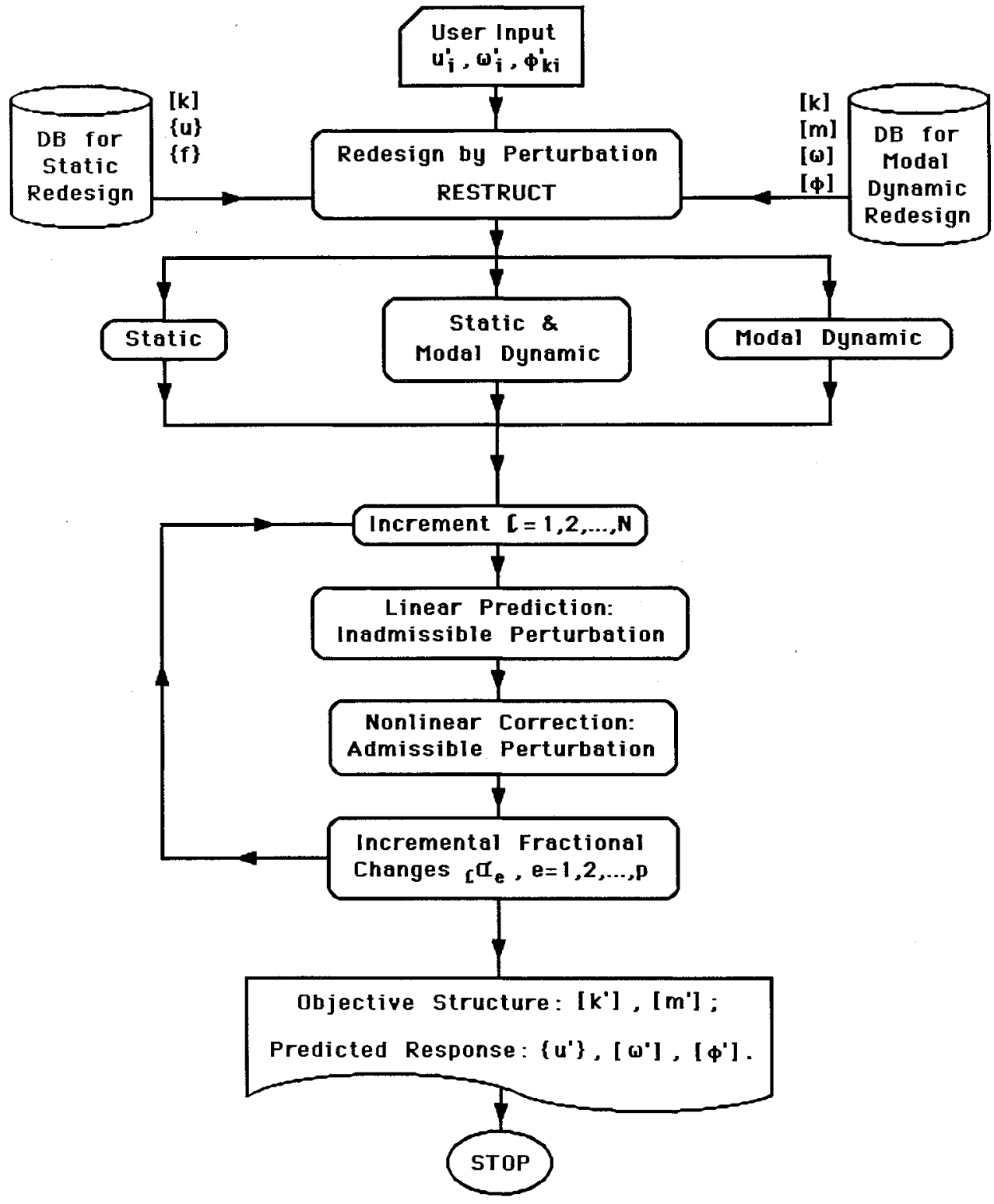

Fig. 2 Structural redesign by RESTRUCT.

case of nonempty continuous feasible domain as

$$
\min \sum_{e=1}^{p}\left[\left(1+\alpha_{e}\right) \prod_{q=1}^{p-1}\left(1+{ }_{q} \alpha_{e}\right)-1\right]^{2}
$$

subject to

$$
\begin{gathered}
u_{i}^{\prime}={ }_{\ell} u_{i}+\delta_{\ell} u_{i} \varliminf_{p} b_{u_{i}}, \quad i=1,2, \ldots, S_{u} \\
\omega_{i}^{\prime 2}={ }_{\ell} \omega_{i}^{2}+\delta_{p} \omega_{i}^{2} \varliminf_{p} b_{\omega_{i}}, \quad i=1,2, \ldots, S_{\omega} \\
\phi_{k i}^{\prime}={ }_{p} \phi_{k i}+\delta_{\ell} \phi_{k i} \varliminf_{p} b_{\phi_{k i}}, \quad \text { number of }(k, i)=S_{\phi} \\
0<\frac{1+\alpha_{e}^{-}}{\prod_{q=1}^{p-1}\left(1+{ }_{q} \alpha_{e}\right)} \leq 1+{ }_{\ell} \alpha_{e} \leq \frac{1+\alpha_{e}^{+}}{\prod_{q=1}^{\ell-1}\left(1+{ }_{q} \alpha_{e}\right)}
\end{gathered}
$$

where $\Pi_{q=1}^{\ell-1}\left(1+{ }_{q} \alpha_{e}\right)$ is known from all previous increments and is by definition one for $\ell=1$; the optimization criterion of Eq. (25) is selected so that at the end of the incremental procedure and completion of the solution process, Eq. (24) is satis- fied since

$$
\left(1+\alpha_{e}\right)=\prod_{\ell=1}^{N}\left(1+{ }_{\ell} \alpha_{e}\right)
$$

$\delta_{\rho} u_{i}, \delta_{\ell} \omega_{i}^{2}, \delta_{\ell} \phi_{k i}$ satisfy the general perturbation equations:

$$
\begin{aligned}
& \ell\{\delta u\}=-\sum_{e=1}^{p}\left({ }_{\ell-1}[k]+\sum_{e=1}^{p}{ }_{\ell-1}\left[k_{e}\right]_{\ell} \alpha_{e}\right)^{-1}{ }_{\ell-1}\left[k_{e}\right]_{\ell-1}\{u\}_{\ell} \alpha_{e} \text { (30) } \\
& \sum_{e=1}^{p}\left(\rho\left\{\psi^{\prime}\right\}_{i \ell-1}^{T}\left[k_{e}\right]_{\ell}\left\{\psi^{\prime}\right\}_{i}-{ }_{\ell} \omega_{i}^{\prime 2} \ell\left\{\psi^{\prime}\right\}_{i p-1}\left[m_{e}\right]_{\ell}\left\{\psi^{\prime}\right\}_{i}\right)_{\ell} \alpha_{e} \\
& ={ }_{p} \omega_{i}^{\prime 2} p\left\{\psi^{\prime}\right\}_{i \ell-1}^{T}[m]_{\ell}\left\{\psi^{\prime}\right\}_{i}-p\left\{\psi^{\prime}\right\}_{i p-1}^{T}[k]_{\ell}\left\{\psi^{\prime}\right\}_{i} \\
& i=1,2, \ldots, n_{r} \\
& \sum_{e=1}^{p}{ }_{\ell}\left\{\psi^{\prime}\right\}_{j \ell-1}^{T}\left[k_{e}\right]_{\ell}\left\{\psi^{\prime}\right\}_{i \ell} \alpha_{e}=-\ell_{\ell}\left\{\psi^{\prime}\right\}_{j \ell-1}^{T}[k]_{\ell}\left\{\psi^{\prime}\right\}_{i} \\
& \sum_{e=1}^{p}\left\{\left\{\psi^{\prime}\right\}_{j \ell-1}^{T}\left[m_{e}\right]_{\ell}\left\{\psi^{\prime}\right\}_{i \ell} \alpha_{e}=-{ }_{\ell}\{\psi\}_{j \ell-1}^{T}[m]_{\ell}\{\psi\}_{j}\right. \\
& \text { for } i=1,2, \ldots, S_{\omega}, \quad j=i+1, i+2, \ldots, n_{r}
\end{aligned}
$$


$n_{r}$ is the number of extracted modes used in the actual redesign computations instead of the total number $n$ of available modes from the baseline eigenanalysis; and primed quantities (objective values) in increment $\ell$ are equal to unprimed quantities (baseline values) in increment $(\ell+1)$.

In each increment, a predictor phase, which is linear in the sense that structural perturbations and allowable changes are small, is first used. Then, a corrector phase is employed to correct those predictions nonlinearly.

\section{Predictor Phase}

This follows the linearization, small-perturbation method proposed by Stetson ${ }^{2-5}$ and extended by Sandström. ${ }^{6,7}$ In that method, the general modal perturbation Eq. (14) is linearized assuming that

$$
\left[\delta_{p} \phi\right]={ }_{\ell}[\phi]_{\ell}[c]^{T}
$$

where ${ }_{\rho}[c]$ is matrix of admixture coefficients with ${ }_{\rho} c_{i i}=0$ and ${ }_{p} c_{i j}, i, j=1,2, \ldots, n_{r}$ are small. Thus, diagonal and off-diagonal terms in Eq. (14) are decoupled. It should be pointed out, however, that in this step the off-diagonal energy balance terms in Eq. (14) are satisfied while admissibility conditions of Eqs. (32) and (33) are violated. Therefore, the conditions satisfied are necessary but not sufficient. In the general case of nonempty continuous feasible domain, the predictor phase redesign problem becomes minimization of Eq. (25) subject to

$$
\begin{aligned}
& \delta_{\ell} \omega_{i}^{2}=\frac{1}{{ }_{\ell} M_{i}}\left[\sum _ { e = 1 } ^ { p } \left({ }_{\ell}\{\psi\}_{i \ell-1}^{T}\left[k_{e}\right]_{\ell}\{\psi\}_{i}\right.\right. \\
& \left.\left.-{ }_{\ell} \omega_{i \ell}^{2}\{\psi\}_{i}^{T}{ }_{\ell-1}\left[m_{e}\right]_{\ell}\{\psi\}_{i}\right)_{\ell} \alpha_{e}\right] \varliminf_{{ }_{\ell}} b_{\omega_{i}}-{ }_{\ell} \omega_{i}^{2} \\
& i=1,2, \ldots, S_{\omega} \\
& \delta_{\ell} \phi_{k i}=\sum_{j=1, \neq i}^{n_{r}} \phi_{k j} c_{i j} \varliminf_{\ell} b_{\phi_{k i}}-{ }_{\ell} \phi_{k i} \\
& -0.1 \leq{ }_{\ell} \alpha_{e} \leq 0.1, \quad \ell=1,2, \ldots, N, \quad e=1,2, \ldots, p
\end{aligned}
$$

and the inequalities of Eq. (29), where lower and upper bounds are imposed on the $\alpha_{e}$ by Eq. (37), as an additional precaution to ensure small perturbations, and

$$
\begin{aligned}
{ }_{\ell} c_{i j} & =\sum_{e=1}^{p} \frac{1}{{ }_{\ell} M_{j}\left({ }_{\ell} \omega_{i}^{2}-{ }_{\ell} \omega_{j}^{2}\right)}\left({ }_{\ell}\{\psi\}_{j \ell-1}^{T}\left[k_{e}\right]_{\ell}\{\psi\}_{i}\right. \\
& \left.-{ }_{\ell} \omega_{i \ell \ell}^{2}\{\psi\}_{j \ell-1}^{T}\left[m_{e}\right]_{\ell}\{\psi\}_{i}\right)_{\ell} \alpha_{e}
\end{aligned}
$$

At the end of the predictor phase, the objective design extracted modes are computed using Eq. (34). Of course this refers only to degrees of freedom of modes not defined by equality constraint of Eq. (36).

\section{Corrector Phase}

In this phase, the predicted eigenmodes are used to correct the values of predicted $\alpha_{e}$. The redesign problem becomes the minimization of Eq. (25) subject to Eqs. (26), (27), (29), and (37); the nonlinear perturbation modal Eq. (31) for $i=j$; the admissibility Eqs. (32) and (33) for $i \neq j$; and the following linear approximation to the static perturbation Eq. $(30)^{18}$;

$$
{ }_{\ell} u_{i}^{\prime}=\sum_{m=1}^{n_{r}} \frac{{ }_{\ell} \phi_{i m \ell}^{\prime} A_{m}}{{ }_{\ell} B_{m}}-\sum_{e=1}^{p}\left(\sum_{m=1}^{n_{r}} \frac{{ }_{\ell} \phi_{i m \ell}^{\prime} A_{m}}{{ }_{\ell} B_{m}^{2}}{ }_{\ell} C_{m e}\right){ }_{\ell} \alpha_{e}
$$

where

$$
\begin{gathered}
{ }_{\ell} A_{m}=\sum_{j=1}^{n}{ }_{\ell} \phi_{j m}^{\prime} f_{j}, \quad{ }_{\ell} B_{m}={ }_{\ell}\left\{\psi^{\prime}\right\}_{m_{\ell-1}}^{T}[k]_{\ell}\left\{\psi^{\prime}\right\}_{m} \\
{ }_{\ell} C_{m e}={ }_{\ell}\left\{\psi^{\prime}\right\}_{m_{\ell-1}}^{T}\left[k_{e}\right]{ }_{\ell}\left\{\psi^{\prime}\right\}_{m}
\end{gathered}
$$

\section{Solvers}

In both predictor and corrector phases, the redesign problem results in an optimization problem with quadratic criterion and linear constraints, which is solved using quadratic programming ${ }^{21}$ and QPSOL. ${ }^{22}$ If the problem has no feasible solution, a generalized inverse algorithm ${ }^{14}$ is used to produce a minimum error solution. RESTRUCT informs the designer accordingly but proceeds with the computations since in subsequent increments feasible solutions may exist.

\section{Computer Implementation}

The redesign process using RESTRUCT is summarized in Fig. 2. The algorithm described in this section is summarized in Fig. 3 and implemented in code RESTRUCT. Several numerical examples are used in the next section to study the efficiency of the new algorithm and its accuracy with respect to previous algorithm that employed inadmissible perturbations. The effect of the number of admissibility constraints and their relaxation on redesign accuracy is also studied by reanalyzing redesigns produced by RESTRUCT using MSC/NASTRAN.

\section{Redesigning in Admissible Domain}

The new algorithm is put into a test by comparing it numerically to a previously developed nonlinear perturbation algorithm that does not force orthogonality conditions on the eigenvectors of the objective structure or any of the intermediate redesigns in the incremental procedure. Accuracy and limitation of the algorithm are assessed by systematic numerical applications using a simple clamped-hinged beam and a 192 DOF offshore tower. Redesigns with as much as $200 \%$ change in response particulars are performed successfully, while emphasis is given to definition and relaxation of admissible domain.

\section{Clamped-Hinged Beam}

The five element, $9 \mathrm{DOF}$, clamped-hinged beam shown in Fig. 4 was first analyzed statically and dynamically using MSC/NASTRAN. First natural frequency and static displacement of the fourth node were computed as $f_{1}=\omega_{1} / 2 \pi=29.14$ $\mathrm{Hz}$ and $u_{4}=7.09 \mathrm{~mm}$, respectively. Eight redesigns are performed and results are summarized in Table 1. The columns titled "Prediction" show the results computed by RESTRUCT. The columns titled "Reanalysis" show the results computed by MSC/NASTRAN for the objective structure provided by RESTRUCT. The relative percentage error is also shown for each case. In all cases, the cross-sectional area and moment of inertia of each one of the five beam elements are allowed to change; that is, ten allowable changes (redesign variables, $p=10$ ) are used. Five modes are extracted and used in the redesign process, $n_{r}=5$. In all applications, $S_{\omega}=1$, $S_{\phi}=0, S_{u}=1, S_{a}=S_{\omega}\left[2\left(n_{r}-1\right)-S_{\omega}\right]=8$. The numerical accuracy used in QPSOL is $\epsilon_{q}=10^{-12}$. The accuracy used in RESTRUCT in satisfying static constraints-Eq. (26)-and diagonal dynamic constraints-Eq. (27)-is $\epsilon_{D}=10^{-6}$. Offdiagonal terms, which are orthogonality constraints with respect to $[m]$ and $[k]$, are satisfied to $\epsilon_{A}=10^{-6}$. Thus, the tolerance used in RESTRUCT in satisfying the admissibility domain is set equal to that used by QPSOL which is equal to $\sqrt{\epsilon_{q}}=10^{-6}$. In the eight redesign applications summarized in Table 1, changes of $6 \%, 30 \%, 100 \%$, and $200 \%$ in equality or inequality redesign goals are used and comparisons are made between the new algorithm that finds an objective structure with admissible modes, and the old algorithm ${ }^{18}$ that forces necessary but not sufficient conditions on the objective structure's modes. For relatively small changes of $6 \%$ and $30 \%$, the two algorithms are about equivalent. For larger changes, the old algorithm becomes less accurate while the new one continues to produce satisfactory results. The mainframe computer of the UB system at The University of Michigan, an IBM 3090 , was used to run all cases and the CPU time in milliseconds is also shown in Table 1. 
INCREMENT L

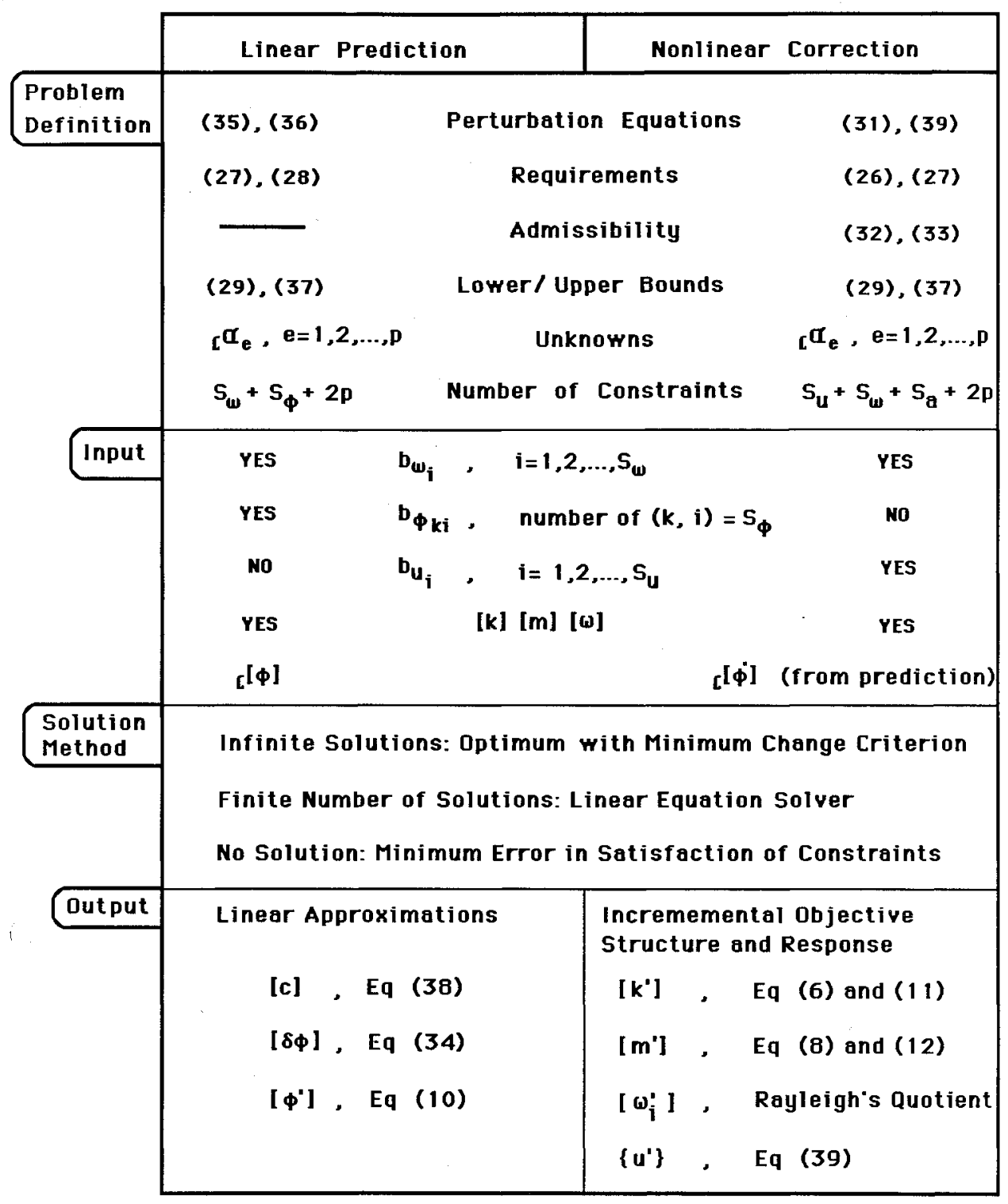

Fig. 3 Algorithm for structural redesign by large admissible perturbations.

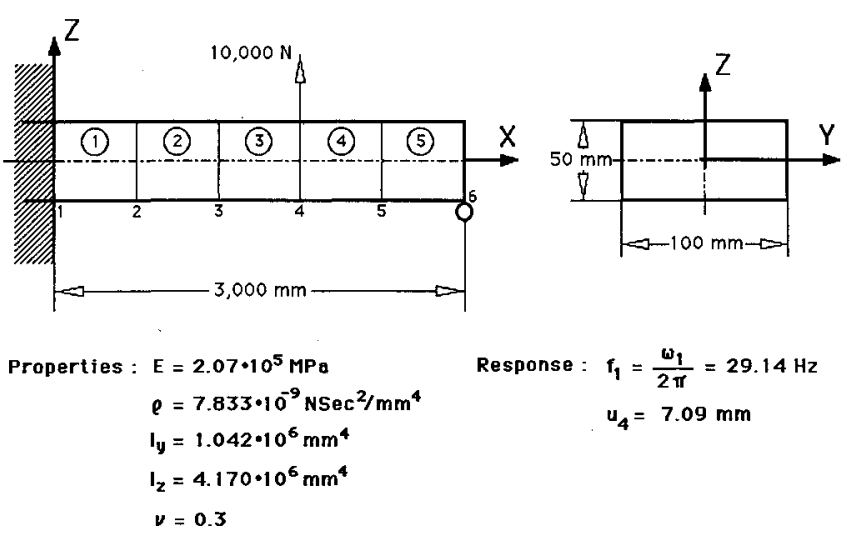

Fig. 4 Five element clamped-hinged beam.

\section{Number of Admissibility Constraints}

The feasibility domain, in which we are looking for the objective structure, is defined by $S_{\omega}+S_{\phi}+S_{u}$ equality or inequality constraints that define the desired response properties of the objective structure, $S_{a}$ admissibility constraints, and $2 p$ upper and lower bounds imposed on redesign variables $\alpha_{e}$, $e=1,2, \ldots, p$. The former are imposed by the designer, and the latter are necessary in order to ensure validity of the linear prediction phase in the incremental algorithm. The admissibility constraints, on the other hand, define whether the eigenmodes of the objective structure correspond to a real structure or not. Their number $S_{a}$ is related to the number of extracted modes by Eq. (22b). The more extracted modes $\left(n_{r}\right)$ are used, the more accurate is the representation of modal dynamics of the baseline structure, the higher is the number of admissibility constraints $S_{a}$, and the more realistic the predicted eigenmodes. Then, the algorithm can be used to redesign structures allowing for larger structural changes. This is demonstrated by the six applications shown in Table 2. It may be expected that the increased number of admissibility constraints may result in numerical complications in the solvers. Our applications, however, do not exhibit such a tendency. In fact, even when the number of equality constraints exceeds the number of unknowns, as in cases 3 and 6 in Table 2 , the accuracy in redesign keeps improving as $S_{\alpha}$ increases. Theoretically, this is expected because once enough orthogonality constraints have been satisfied to produce a unique set of $\alpha_{e}, e=1,2, \ldots, p$, any addi- 
Table 1 Perturbation structural redesign with or without admissibility constraints ${ }^{\text {a }}$

\begin{tabular}{|c|c|c|c|c|c|c|c|c|c|}
\hline \multirow[b]{3}{*}{ Case no. } & \multicolumn{2}{|c|}{ Redesign goals } & \multicolumn{7}{|c|}{ Without admissibility constraints (Old Algorithm) } \\
\hline & \multirow{2}{*}{$\frac{\omega_{1}^{\prime 2}}{\omega_{l}^{2}}$} & \multirow{2}{*}{$\frac{u_{4}^{\prime}}{u_{4}}$} & \multicolumn{3}{|c|}{$\omega_{1}^{\prime 2} / \omega_{1}^{2}$} & \multicolumn{3}{|c|}{$u_{4}^{\prime} / u_{4}$} & \multirow[b]{2}{*}{ CPU, ms } \\
\hline & & & Prediction & Reanalysis & Error, $\%$ & Prediction & Reanalysis & Error, $\%$ & \\
\hline I & 1.06 & 1.06 & 1.060 & 1.0600 & 0.001 & 1.064 & 1.0667 & 0.632 & 1600 \\
\hline 2 & 1.30 & 1.30 & 1.300 & 1.2995 & -0.038 & 1.305 & 1.3156 & 1.20 & 4170 \\
\hline 3 & 2.00 & 2.00 & 2.000 & 1.9922 & -0.392 & 2.007 & 2.0604 & 3.02 & 10050 \\
\hline 4 & 3.00 & 3.00 & 3.000 & 2.9468 & -1.773 & 3.011 & 3.1820 & 6.067 & 15301 \\
\hline 5 & $>1.06$ & $>1.06$ & 1.060 & 1.0604 & Satisfied & 1.064 & 1.0642 & Satisfied & 1617 \\
\hline 6 & $>1.30$ & $>1.30$ & 1.300 & 1.2951 & -0.374 & 1.305 & 1.3071 & Satisfied & 4287 \\
\hline 7 & $>2.00$ & $>2.00$ & 2.000 & 1.9592 & -2.039 & 2.008 & 2.0380 & Satisfied & 10095 \\
\hline \multirow[t]{3}{*}{8} & $>3.00$ & $>3.00$ & 3.000 & 2.8661 & -4.463 & 3.012 & 3.1206 & Satisfied & 16010 \\
\hline & \multicolumn{2}{|c|}{ Redesign goals } & \multicolumn{7}{|c|}{ With admissibility constraints (New Algorithm) } \\
\hline & $\omega_{1}^{\prime 2}$ & $\underline{u_{4}^{\prime}}$ & \multicolumn{3}{|c|}{$\omega_{1}^{\prime} / \omega_{1}^{2}$} & \multicolumn{3}{|c|}{$u_{4}^{\prime} / u_{4}$} & \\
\hline Case no. & $\overline{\omega_{1}^{2}}$ & $\overline{u_{4}}$ & Prediction & Reanalysis & Error, $\%$ & Prediction & Reanalysis & Error, $\%$ & $\mathrm{CPU}, \mathrm{ms}$ \\
\hline 1 & 1.06 & 1.06 & 1.060 & 1.0600 & 0.002 & 1.064 & 1.0654 & $0 . \overline{508}$ & 1408 \\
\hline 2 & 1.30 & 1.30 & 1.300 & 1.2994 & -0.046 & 1.305 & 1.3078 & 0.600 & 4305 \\
\hline 3 & 2.00 & 2.00 & 2.000 & 1.9940 & -0.301 & 2.008 & 2.0168 & 0.840 & 10153 \\
\hline 4 & 3.00 & 3.00 & 3.000 & 2.9744 & -0.853 & 3.0120 & 3.0400 & 1.333 & 15796 \\
\hline 5 & $>1.06$ & $>1.06$ & 1.060 & 1.0600 & Satisfied & 1.064 & 1.0654 & Satisfied & 1419 \\
\hline 6 & $>1.30$ & $>1.30$ & 1.300 & 1.2994 & -0.047 & 1.305 & 1.3078 & Satisfied & 4132 \\
\hline 7 & $>2.00$ & $>2.00$ & 2.000 & 1.9940 & -0.301 & 2.008 & 2.0168 & Satisfied & 10209 \\
\hline 8 & $>3.00$ & $>3.00$ & 3.000 & 2.9744 & -0.853 & 3.0120 & 3.0400 & Satisfied & 16751 \\
\hline
\end{tabular}

Table 2 Effect of number of admissibility constraints on redesign a

\begin{tabular}{|c|c|c|c|c|c|c|c|c|c|c|c|}
\hline \multirow[b]{3}{*}{ Case no. } & \multirow[b]{3}{*}{$n_{r}$} & \multirow[b]{3}{*}{$S_{a}$} & \multicolumn{2}{|c|}{$\begin{array}{l}\text { Redesign } \\
\text { goals }\end{array}$} & \multicolumn{3}{|c|}{$\omega_{1}^{2} / \omega_{l}^{2}$} & \multicolumn{3}{|c|}{$u_{4}^{\prime} / u_{4}$} & \multirow[b]{3}{*}{$\mathrm{CPU}, \mathrm{ms}$} \\
\hline & & & $\omega_{1}^{\prime 2}$ & $\underline{u_{4}^{\prime}}$ & & & & & & & \\
\hline & & & $\omega_{1}^{2}$ & $\overrightarrow{u_{4}}$ & Prediction & Reanalysis & Error, $\%$ & Prediction & Reanalysis & Error, $\%$ & \\
\hline 1 & 3 & 4 & 1.30 & 1.30 & 1.300 & 1.2923 & -0.590 & 1.305 & 1.3291 & 2.240 & 2642 \\
\hline 2 & 5 & 8 & 1.30 & 1.30 & 1.300 & 1.2994 & -0.043 & 1.305 & 1.3078 & 0.600 & 4208 \\
\hline 3 & 8 & 14 & 1.30 & 1.30 & 1.300 & 1.3000 & $3.16 \times 10^{-4}$ & 1.305 & 1.3054 & 0.412 & 7453 \\
\hline 4 & 3 & 4 & 2.00 & 2.00 & 2.000 & 1.8860 & -5.70 & 2.008 & 2.1904 & 9.521 & 6802 \\
\hline 5 & 5 & 8 & 2.00 & 2.00 & 2.000 & 1.9937 & -0.314 & 2.008 & 2.0168 & 0.842 & 11142 \\
\hline 6 & 8 & 14 & 2.00 & 2.00 & 2.000 & 2.0000 & $2.535 \times 10^{-4}$ & 2.008 & 2.0075 & 0.375 & 20153 \\
\hline
\end{tabular}

${ }^{a} p=10, \epsilon_{D}=10^{-6}, \epsilon_{A}=10^{-3}$.

tional orthogonality constraint should be automatically satisfied by that unique objective structure because it is admissible and therefore real. Thus, in cases 3 and 6 , even though the number of admissibility constraints is greater than the number of redesign variables $\left(S_{a}=14 \geq 10=p\right)$, and the generalized inverse algorithm is used to produce a minimum-error solution, the solution is very accurate.

Looking at the above conclusion from another point of view, namely that of relaxation of the admissibility domain, we observe that relaxation results in less accurate answers. Equivalently, fewer admissibility constraints result in less accurate enforcement of orthogonality and limitation of effectiveness of the algorithm to moderate structural changes. An alternate form of relaxation of the admissibility domain is use of greater tolerances-higher values of $\epsilon_{D}$ and $\epsilon_{A}$. This has a lesser impact on the accuracy and limitation of RESTRUCT.

\section{Offshore Tower}

The offshore tower shown in Fig. 5 is $69.95 \mathrm{~m}$ high and operates in $45.72-\mathrm{m}$ water depth. The tower at the base is $38.10 \mathrm{~m}$ square and tapers linearly to $22.86 \mathrm{~m}$ square at the deck. The FE model of the tower is composed of 104 circular tubular beam elements and has 192 degrees of freedom. Loading on the tower is due to 1) 240 tonnes deck load that is applied to the structure as uniformly distributed load at the deck nodal points; 2) wave hydrodynamic forces calculated for a design wave of $182.88 \mathrm{~m}$ length and $6.10 \mathrm{~m}$ height using Morison's equation; the wave propagates in the $x$ direction; 3 ) wind current in the $x$ direction with linear velocity profile of $1.03 \mathrm{~m} / \mathrm{s}$ at the mean free surface waterline and zero at the sea bed.

Static and dynamic analyses by MSC/NASTRAN produced 1) maximum displacement in the $x$ direction at node 11 (shown in Fig. 5) equal to $0.0578 \mathrm{~m}$, and 2) a repeated first natural frequency $\omega_{1}=\omega_{2}=4.695 \mathrm{radian} / \mathrm{s}\left(f_{1}=f_{2}=0.7472 \mathrm{~Hz}\right)$. For manufacturability reasons, in the redesign process the tower is divided into six element groups described in Table 3. Each group changes uniformly. The total number of design variables is $p=12$, that is, two variables are allowed to change in each group of elements. For mass and axial stiffness the area is allowed to change, while for bending and torsional stiffness the area moment of inertia is allowed to change.

Two redesign applications are considered and the results are summarized in Table 4 . In those applications, only three modes were extracted in order to show the accuracy of the redesign algorithm even for such a minimal number of extracted modes. Those modes, however, were selected judiciously. Specifically, since one of the redesign goals is related to $\omega_{1}$, only modes 1,8 , and 9 were extracted. Modes 8 and 9 are the second bending modes in the $(x, z)$ and $(y, z)$ plane, respectively. Those have maximum relation to mode 1 as the corresponding admixture coefficients show. Modes 1 and 2 are highly related, but the latter is excluded because $\omega_{1}=\omega_{2}$ and consequently the admixture coefficients cannot be defined by Eq. (38). This problem of repeated eigenvalues is solved in Ref. 18. Other modes have low relation to mode 1 and consequently do not affect its change. For example, the first torsional mode about the $z$ axis has a low relation to mode 1 , which is the first bending mode in the $(x, z)$ plane. 


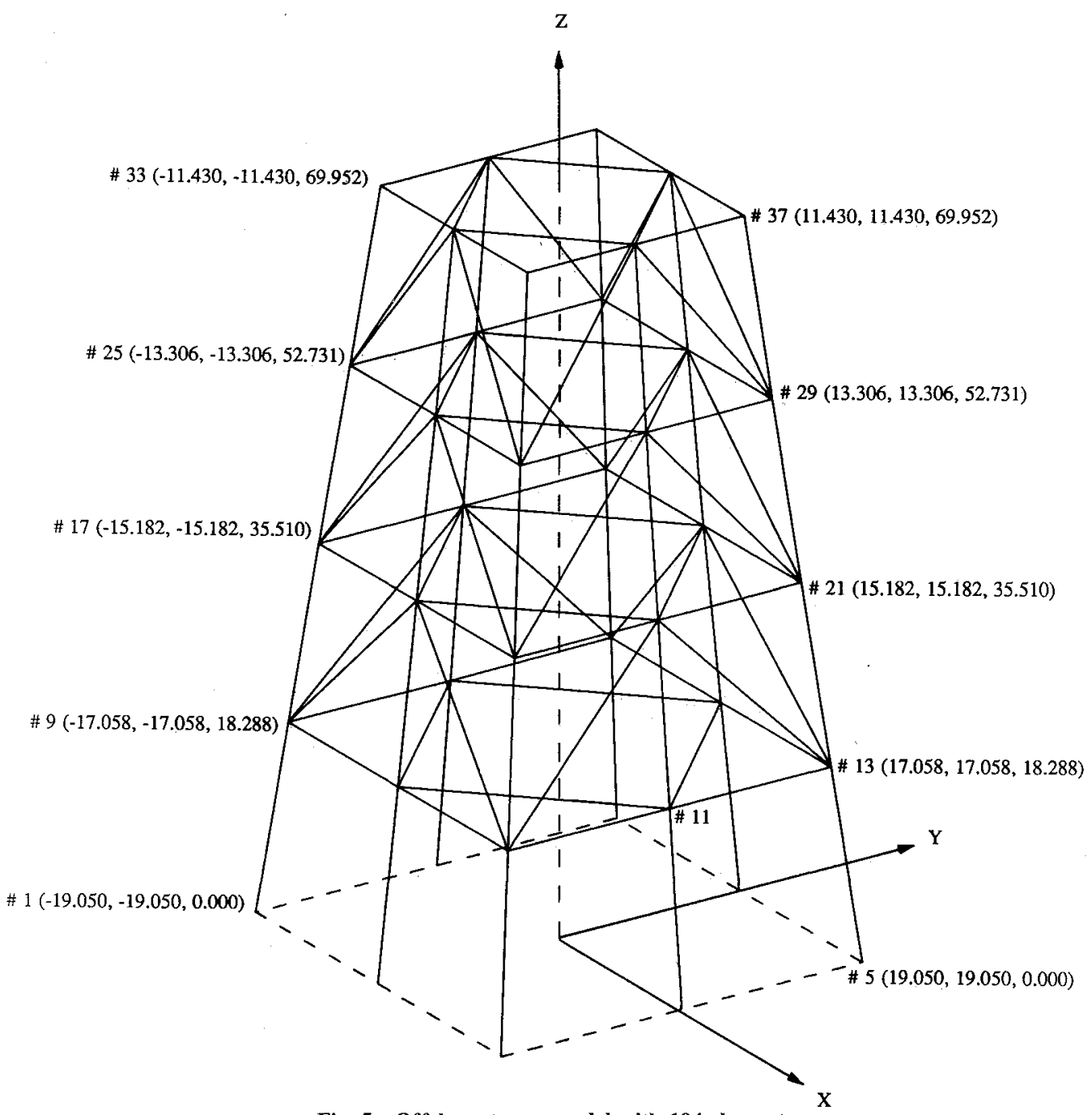

Fig. 5 Offshore tower model with 104 elements.

Table 3 Offshore tower substructures, redesign variables and dimensions

\begin{tabular}{cccccc}
\hline \hline $\begin{array}{c}\text { Substructure } \\
\text { number }\end{array}$ & $\begin{array}{c}\text { Design } \\
\text { variable } \\
\alpha_{e}\end{array}$ & $\begin{array}{c}\text { Substructure } \\
\text { description }\end{array}$ & $\begin{array}{c}D_{o}, \\
\mathrm{~m}\end{array}$ & $\begin{array}{c}D_{i}, \\
\mathrm{~m}\end{array}$ & $\begin{array}{c}\text { Number } \\
\text { of } \\
\text { elements }\end{array}$ \\
\hline 1 & $\begin{array}{c}\alpha_{1}\left(\alpha_{I}\right) \\
\alpha_{2}\left(\alpha_{A}\right)\end{array}$ & $\begin{array}{c}\text { Legs below first } \\
\text { bracing }\end{array}$ & 0.762 & 0.737 & 8 \\
2 & $\begin{array}{l}\alpha_{3}\left(\alpha_{I}\right) \\
\alpha_{4}\left(\alpha_{A}\right)\end{array}$ & $\begin{array}{c}\text { Legs between first } \\
\text { and second } \\
\text { bracing }\end{array}$ & 0.610 & 0.584 & 8 \\
3 & $\begin{array}{l}\alpha_{5}\left(\alpha_{I}\right) \\
\alpha_{6}\left(\alpha_{A}\right)\end{array}$ & $\begin{array}{c}\text { Legs above } \\
\text { second bracing }\end{array}$ & 0.610 & 0.584 & 16 \\
4 & $\begin{array}{l}\alpha_{7}\left(\alpha_{I}\right) \\
\alpha_{8}\left(\alpha_{A}\right)\end{array}$ & $\begin{array}{c}\text { Horizontal } \\
\text { bracing }\end{array}$ & 0.483 & 0.464 & 32 \\
5 & $\begin{array}{l}\alpha_{9}\left(\alpha_{I}\right) \\
\alpha_{10}\left(\alpha_{A}\right)\end{array}$ & $\begin{array}{c}\text { Horizontal cross } \\
\text { bracing }\end{array}$ & 0.508 & 0.489 & 16 \\
6 & $\begin{array}{l}\alpha_{11}\left(\alpha_{I}\right) \\
\alpha_{12}\left(\alpha_{A}\right)\end{array}$ & $\begin{array}{c}\text { Vertical cross } \\
\text { bracing }\end{array}$ & 0.610 & 0.591 & 24 \\
\hline \hline
\end{tabular}

\section{Conclusions}

Nonlinear structural redesign methods that are based on perturbation allow for large changes between baseline and objective structures, produce the desired redesign without trial and error, and require only one finite element analysis that of the baseline structure. Redesign objectives may be any combination of modal properties-natural frequencies and modal shapes - and static displacements. The derived general perturbation equations are implicit strongly nonlinear equation of the allowable structural changes. To solve them, an incremental algorithm with prediction-correction is developed which employs only admissible structural changes. Admissibility of structural changes is defined as the condition where at each increment the modes computed by perturbation correspond to a real structure; i.e., they satisfy the orthogonality conditions with respect to the mass and stiffness matrices. This implies that necessary and sufficient orthogonality conditions are enforced numerically. The new algorithm successfully redesigns structures that require very large changes in their

Table 4 Large perturbation admissible redesign of offshore tower ${ }^{a}$

\begin{tabular}{|c|c|c|c|c|c|c|c|c|c|}
\hline \multirow[b]{3}{*}{ Case no. } & \multicolumn{2}{|c|}{$\begin{array}{c}\text { Redesign } \\
\text { goals }\end{array}$} & \multicolumn{3}{|c|}{$\omega_{1}^{\prime 2} / \omega_{1}^{2}$} & \multicolumn{3}{|c|}{$u_{11}^{\prime} / u_{11}$} & \multirow[b]{3}{*}{$\mathrm{CPU}, \mathrm{ms}$} \\
\hline & $\omega_{1}^{\prime 2}$ & $\underline{u_{11}^{\prime}}$ & & & & & & & \\
\hline & $\overline{\omega_{1}^{2}}$ & $\overline{u_{11}}$ & Prediction & Reanalysis & Error, $\%$ & Prediction & Reanalysis & Error, $\%$ & \\
\hline 1 & 1.50 & 1.50 & 1.500 & 1.4715 & -1.426 & 1.507 & 1.5379 & 2.526 & 433487 \\
\hline 2 & 2.00 & 2.00 & 2.000 & 1.8916 & -5.421 & 2.008 & 2.1036 & 5.18 & 784491 \\
\hline
\end{tabular}


modal dynamic and static displacement response. Systematic numerical applications are used to study the accuracy and limitations of RESTRUCT using a simple clamped-hinged beam and an offshore tower. Successful redesigns that required as much as $200 \%$ changes in their response characteristics were performed.

\section{Acknowledgments}

This publication has been sponsored by the University of Michigan/Sea Grant/Industry Consortium in Off́shore Engineering under Michigan Sea Grant College Program, projects E/GLE-14 and R/T-23, under Grant NA85AA-D-SG045C from the Office of Sea Grant, National Oceanic and Atmospheric Administraion (NOAA), U.S. Department of Commerce, and funds from the State of Michigan. Industry participants include the American Bureau of Shipping; ARCO Gas and Oil Company (1988-); Conoco, Inc.; Exxon Production Research; Friede and Goldman, Ltd.; Noble, Denton and Associates, Inc. (1985 1986); Shell Companies Foundation (1985-1986); and the U.S. Coast Guard (1986-). The U.S. Government is authorized to produce and distribute reprints for governmental purpose notwithstanding any copyright notation appearing hereon.

\section{References}

${ }^{1}$ Haftka, R. T., and Prasad, B., "Programs for Analysis and Resizing of Complex Structures," Computers and Structures, Vol. 10, No. 2, 1979, pp. 323-330.

${ }^{2}$ Haug, E. J., Choi, K. K., and Komkov, V., Design Sensitivity Analysis of Structural Systems, Academic, Orlando, FL, 1986, Chap. 1.

${ }^{3}$ Choi, K. K., Haug, E. J., and Seong, H. G., "An Iterative Method for Finite Dimensional Structural Optimization Problems with Repeated Eigenvalues," International Journal for Numerical Methods in Engineering, Vol. 19, No. 1, 1983, pp. 93-112.

${ }^{4}$ Stetson, K. A., "Perturbation Method of Structural Design Relevant to Holographic Vibration Analysis," AIAA Journal, Vol. 13, No. 4, 1975, pp. 457-459.

${ }^{5}$ Stetson, K. A., and Palma, G. E., "Inversion of First-Order Perturbation Theory and Its Application to Structural Design," AIAA Journal, Vol. 14, No. 4, 1976, pp. 454-460.

${ }^{6}$ Stetson, K. A., Harrison, I. R., and Palma, G. E., "Redesigning Structural Vibration Modes by Inverse Perturbation Subject to Minimal Change Theory," Computer Methods in Applied Mechanics and Engineering, Vol. 16, No. 2, Nov. 1978, pp. 151-175.

${ }^{7}$ Stetson, K. A., and Harrison, I. R., " Redesign of Structural Vibration Modes by Finite-Element Inverse Perturbation," ASME Transactions, Journal of Engineering for Power, Vol. 103, No. 2, 1981, pp. 319-325.
${ }^{8}$ Sandström, R. E., "Inverse Perturbation Methods for Vibration Analysis," Proceedings, NATO Advance Study Institute of Optimization of Distributed Parameter Structural Systems, University of Iowa, May 20-June 4, 1980.

${ }^{9}$ Sandström, R. E., and Anderson, W. J., "Modal Perturbation Methods for Marine Structures," Transactions, Society of Naval Architecture and Marine Engineering, Vol. 90, Nov. 1982, pp. 41-54.

${ }^{10} \mathrm{Kim}, \mathrm{K}$. O., Anderson, W. J., and Sandström, R. E., "Nonlinear Inverse Perturbation Method in Dynamic Analysis," AIAA Journal, Vol. 21, No. 9, 1983, pp. 1310-1316.

${ }^{11} \mathrm{Kim}, \mathrm{K} . \mathrm{O}$., and Anderson, W. J., "Dynamic Condensation in Structural Dynamics Redesign," AIAA Journal, Vol, 22, No. 11, 1984, pp. 1616-1617.

${ }^{12}$ Hoff, C. J., Bernitsas, M. M., Sandström, R. E., and Anderson, W. J., "Nonlinear Incremental Inverse Perturbation Method for Structural Redesign," AIAA Journal, Vol. 22, No. 9, 1984, pp. 13041309.

${ }^{13}$ Hoff, C. J., and Bernitsas, M. M., "Nonlinear Inverse Perturbation Methods in Structural Dynamics Redesign of Offshore Structures," Proceedings of the 3rd International Congress on Marine Technology, Athens '84, International Maritime Association of East Mediterranean, Athens, Greece, May 28-June 1, 1984, pp. 311-320.

${ }^{14}$ Hoff, C. J., and Bernitsas, M. M., "Dynamic Redesign of Marine Structures," Journal of Ship Research, Vol. 29, No. 4, 1985, pp. 285295.

${ }^{15}$ Bernitsas, M. M., Hoff, C. J., and Kokarakis, J. E., "Nonlinear Inverse Perturbation in Structural Redesign of Risers," ASME Transactions, Journal of Energy Resources Technology, Vol. 107, No. 2, 1985 , pp. 256-263.

${ }^{16}$ Gans, H. D., and Anderson, W. J., "Structural Optimization Incorporating Centrifugal and Coriolis Effects," Proceedings of 30th AIAA/ASME/ASCE/AHS/ASC Structures, Structural Dynamics, and Materials Conference, AIAA, Washington, DC, April 3-5, 1989, pp. 1311-1320.

${ }^{17}$ Hoff, C. J., and Bernitsas, M. M., "Static Redesign of Offshore Structures," Proceedings, 5th International OMAE Symposium, Vol. III, ASME, New York, 1986, pp. 78-85.

${ }^{18} \mathrm{Kim}$, J. H., and Bernitsas, M. M., "Redesign of Marine Structures," Journal of Marine Structures, Vol. 1, No. 2, 1988, pp. 139183.

${ }^{19}$ Bernitsas, M. M., and Kim, J., "RESTRUCT: A Program for Redesign of Structures," Rept. to The Univ. of Michigan/Sea Grant/ Industry Consortium in Offshore Engineering and Publication 303, Dept. of Naval Architecture and Marine Engineering, The Univ. of Michigan, Ann Arbor, MI, Sept. 1987.

${ }^{20}$ Baldwin, J. F., and Hutton, S. G., "Natural Modes of Modified Structures," AIAA Journal, Vol. 23, No. 11, 1985, pp. 1737-1743.

${ }^{21}$ Luenberger, D. G., Introduction to Linear and Nonlinear Programming, Addison-Wesley, Reading, MA, 1973, Chap. 3.

${ }^{22}$ Gill, P. E., Murray, W., Saunders, M. A., and Wright, M. H., "User's Guide for SOL/QPSOL: A Fortran Package for Quadratic Programming," Dept. of Operations Research, Stanford Univ., Stanford, CA, 1983. 